\title{
Estrategias de forrajeo de Robsonella fontaniana (d'Orbigny, 1834) (Cephalopoda: Octopodidae)
}

\author{
Feeding strategies of Robsonella fontaniana (d’Orbigny, 1834) (Cephalopoda: Octopodidae)
Christian M. Ibáñez ${ }^{1}$, Roger D. Sepúlveda², Evelin Sanhueza ${ }^{3}$, J. Francisco Ruiz ${ }^{4}$ y Javier Chong ${ }^{5}$

\author{
${ }^{1}$ Instituto de Ecología y Biodiversidad, Departamento de Ciencias Ecológicas, Facultad de Ciencias, Universidad de Chile, \\ Casilla 653, Santiago, Chile \\ ${ }^{2}$ Instituto de Ecología y Evolución, Facultad de Ciencias, Universidad Austral de Chile, Casilla 567, Valdivia, Chile \\ ${ }^{3}$ Instituto de Investigación Pesquera, Avenida Cristóbal Colón 2780, Talcahuano, Chile \\ ${ }^{4}$ Departamento de Biología Marina, Facultad de Ciencias del Mar, Universidad Católica del Norte, Casilla 117, Coquimbo, Chile \\ ${ }^{5}$ Departamento de Ecología Costera, Facultad de Ciencias, Universidad Católica de la Santísima Concepción, \\ Casilla 297, Concepción, Chile \\ ibanez.christian@gmail.com
}

\begin{abstract}
In this study we analyzed the natural diet and the dietary preferences of the octopus Robsonella fontaniana (d'Orbigny, 1834). Twenty-seven individuals were collected at San Vicente Bay, Chile, to study their stomach contents, and other 12 individuals were used in laboratory experiments for dietary selection tests. In the stomach contents 11 prey types were found, from which brachyuran crabs were the most frequent item. For preference tests, the animals were acclimated to constant temperature, air and water. Two experiments were performed: i) selection test on two gastropod species and three crustacean species, resulting in consume of crustaceans only (66.7\% crustaceans and $0 \%$ gastropods), and ii) selection test on five crustaceans species, showing high preference for those highly motile (i.e. E. analoga was consumed $75 \%$ of the times offered). The scaling relationship between octopuses and preys showed a positive relationship between the size of octopuses and the fed preys. From field and experimental evidence we suggest that $R$. fontaniana has a selective hunting behavior and specialized diet with a narrow trophic niche breadth.
\end{abstract}

Key words: Predation, feeding, octopuses, opportunistic predators
Resumen.- En este estudio se analizó la dieta natural y las preferencias dietarias del pulpo Robsonella fontaniana (d’Orbigny, 1834). Veintisiete individuos fueron recolectados en Bahía San Vicente, Chile, para estudiar sus contenidos estomacales, y otros 12 individuos fueron usados en experimentos de laboratorio para pruebas de selección dietaria. En el contenido estomacal se encontraron 11 tipos de presas, de los cuales los crustáceos braquiuros fueron los ítemes presa más frecuentes. Para las pruebas de preferencia, los animales fueron aclimatados a condiciones constantes de temperatura, aire y agua. Se realizaron dos experimentos: i) pruebas de selección sobre dos especies de gasterópodos y tres especies de crustáceos, resultando en consumo sólo de crustáceos (66,7\% de crustáceos y $0 \%$ de gasterópodos) y ii) pruebas de selección sobre cinco especies de crustáceos, mostrando alta preferencia por aquellas altamente móviles (i.e. E. analoga fue consumida el $75 \%$ de las veces ofrecidas). La relación de tamaño entre pulpos y presas mostró una relación positiva entre el tamaño de los pulpos y las presas consumidas. A partir de la evidencia de terreno y laboratorio, sugerimos que $R$. fontaniana presenta una conducta de caza selectiva y una dieta especializada representada por un nicho trófico restringido.

Palabras clave: Depredación, alimentación, pulpos, depredadores oportunistas

\section{Introducción}

Los depredadores no solo matan y consumen sus presas sino que también presentan variadas conductas y estrategias de forrajeo relacionadas con las características fenotípicas y ecológicas de sus presas. Consecuentemente, los depredadores se pueden clasificar en oportunistas y/o selectivos, los cuales han sido confundidos por los equivalentes de generalista vs. especialista, o la de minimizador de tiempo vs. maximizador de energía (Jaksic \& Marone 2007). Todas estas dicotomías hacen referencia a diferentes atributos fenotípicos de los depredadores relacionados con la conducta y tácticas de caza como también con la dieta y amplitud del nicho trófico (Jaksic \& Marone 2007). 
Los pulpos bentónicos de la familia Octopodidae son depredadores y generalmente tienen un amplio nicho trófico, por lo que han sido clasificados erradamente como depredadores oportunistas (Ambrose \& Nelson 1983, Iribarne et al. 1991, Rodhouse \& Nigmatullin 1996, Oosthuizen \& Smale 2003, Cardoso et al. 2004). Alternativamente, algunos autores han señalado correctamente que la dieta depende de la abundancia de las presas disponibles en el ambiente circundante (Guerra 1978, Smale \& Bucham 1981, Ambrose 1986, Vincent et al. 1998). Sin embargo, experimentos de campo y laboratorio han demostrado cierto grado de selectividad de las presas consumidas por los pulpos, relacionado principalmente con la capacidad de manipulación y el tamaño corporal de las presas (Fiorito \& Gherardi 1999, Anderson \& Mather 2007, Anderson et al. 2008). Estudios de campo han señalado que la dieta de los pulpos depende, entre otros factores, de la disponibilidad de hábitat, estacionalidad, tamaño corporal y madurez sexual (e.g. Smale \& Bucham 1981, Cortez et al. 1995, Smith 2003, Rosa et al. 2004, Ibáñez \& Chong 2008), siendo el tamaño corporal la variable más importante para explicar las variaciones en la composición de la dieta (Ibáñez \& Chong 2008). Por esta razón en algunos estudios se ha encontrado que tanto la composición, el tamaño y la diversidad de presas se relacionan con el tamaño de los pulpos (Smale \& Bucham 1981, Steer \& Semmens 2003, Smith 2003, Ibáñez \& Chong 2008). Estos factores (ecológicos y fenotípicos), asociados a los tiempos de eficiencia de forrajeo de los pulpos sobre una determinada especie de presa, son una fuente de variación importante a la hora de determinar los patrones conductuales de alimentación, tanto inter- como intra-específicos (Forsythe \& Hanlon 1997).

Robsonella fontaniana (d’Orbigny, 1834) es un pulpo bentónico de pequeño tamaño corporal que se distribuye desde el norte del Perú hasta el Golfo Nuevo en Argentina pasando por el cono sur, incluyendo toda la costa de Chile (Ibáñez et al. 2008). Esta especie habita la zona intermareal hasta los 225 metros de profundidad sobre sustratos duros, y en menor abundancia sobre sustratos arenosos (Ibáñez et al. 2008). Poco se conoce acerca de su biología y ecología debido a que no es muy abundante y no constituye un recurso pesquero importante en las costas de Sudamérica. Sin embargo, es una especie muy interesante y útil como modelo de estudio para poner a prueba hipótesis sobre las estrategias de forrajeo de este tipo de depredadores.

Este trabajo tiene como objetivo describir la alimentación natural y determinar las preferencias alimenticias en condiciones experimentales del pulpo $R$. fontaniana, poniendo a prueba la hipótesis de que los pulpos presentan una estrategia de forrajeo oportunista debido a su amplio nicho trófico (dieta generalizada).

\section{Material y métodos}

\section{Análisis del contenido estomacal}

Un total de 27 individuos de $R$. fontaniana fueron obtenidos mediante buceo autónomo en la localidad de Lenga (Bahía San Vicente, Chile, 3644'S, 73¹0’W) de enero a marzo de 2003. A cada individuo se le registró la longitud dorsal del manto (LM, cm), el peso total (PT, g) y se les determinó el sexo. El estómago de cada individuo fue extraído, congelado a $-20^{\circ} \mathrm{C}$ y posteriormente analizado para identificar las presas consumidas lo cual se realizó mediante literatura especializada en crustáceos (Retamal 1981) y colecciones de referencia. El nivel taxonómico de las presas fue condicionado por el grado de digestión que presentó el contenido estomacal, y debido a esto, sólo se pudo calcular la frecuencia de ocurrencia relativa $(\% \mathrm{~F})$ de cada presa en cada contenido estomacal. Con los datos de frecuencia se calculó el índice B' de amplitud de nicho trófico (Cardona 1991), el cual va desde 0,01 (dieta especializada) a 1,00 (dieta generalizada).

\section{Experimentos de preferencia alimenticia}

Un grupo de experimentos fue realizado para probar la capacidad del pulpo para seleccionar especies como presas siguiendo a Iribarne et al. (1991). Todos los pulpos y sus potenciales presas fueron obtenidos de Lenga, Bahía San Vicente, en febrero de 2003. Los 12 ejemplares fueron colocados en acuarios de acondicionamiento por $24 \mathrm{~h}, \mathrm{y}$ luego trasladados a acuarios de $100 \mathrm{~L}$ por otras $24 \mathrm{~h}$, con temperatura ambiente, aireación y flujo de agua de mar constante del sitio de estudio. Los ejemplares fueron mantenidos sin alimentación durante $48 \mathrm{~h}$. Las potenciales presas fueron capturadas al azar en el intermareal rocoso aledaño al laboratorio costero en Lenga, por lo tanto el tamaño de las presas ofrecidas también fue azaroso (entre 14 y 27 mm de diámetro para los gasterópodos y entre 11 y 30 mm de longitud de cefalotórax para los crustáceos). En todos los casos los pulpos fueron mantenidos en forma individual para luego probar los siguientes tratamientos por 24 horas:

1) Selectividad de presas entre crustáceos y gasterópodos, realizado con nueve individuos de $R$. fontaniana. A cada pulpo se le ofrecieron como presas potenciales cinco individuos de las siguientes especies: los gasterópodos Tegula atra (Lesson, 1830) y Diloma nigerrima (Gmelin, 1791), y los crustáceos Petrolisthes laevigatus (Guérin, 1835), Cyclograpsus cinereus (Dana, 1851) y Hemigrapsus crenulatus (Milne-Edwards, 1837). 
2) Selectividad de presas entre especies de crustáceos, realizado con 12 individuos de $R$. fontaniana. A cada pulpo se le ofrecieron cinco individuos como presas potenciales de las siguientes especies: los braquiuros Cancer coronatus (Molina, 1782), Cyclograpsus cinereus, Hemigrapsus crenulatus, y los anomuros Petrolisthes laevigatus y Emerita analoga (Stimpson, 1857).

Pulpos y presas potenciales fueron medidos y pesados antes y después de cada experimento para calcular el peso del alimento consumido. Todas las presas fueron colocadas en un sub-acuario dividido en espacios más pequeños. Ambos experimentos fueron realizados en fondo duro (acuario), y las frecuencias resultantes fueron evaluadas con tablas de contingencia a través del estadígrafo Chi-cuadrado (Zar 1999).

Adicionalmente, en los experimentos de laboratorio se estudiaron las relaciones entre la longitud del manto de los pulpos y la longitud de presas, el peso total de los pulpos y el peso del alimento consumido, y el peso total de los pulpos y el porcentaje del alimento consumido en relación al peso de los pulpos. Todas estas relaciones fueron evaluadas a través del cálculo del coeficiente de correlación de Spearman (Zar 1999). Debido al bajo tamaño muestreal, la significancia estadística de esta correlación fue estimada a través de una prueba de permutaciones con $n=1000$ iteraciones (sensu Hammer et al. 2001).

\section{Resultados}

En el 74\% de los estómagos de los 27 pulpos capturados se encontró contenido estomacal, los que correspondieron en su mayoría a machos (78\%). La longitud del manto de los pulpos fluctuó entre 25 y $65 \mathrm{~mm}(44,1 \pm 9,9 \mathrm{~mm}$ promedio \pm desviación estándar) y su peso total entre 4,9

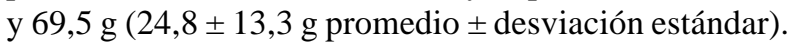

El contenido estomacal de los pulpos se encontraba en un estado muy digerido y las presas fueron muy difíciles de identificar taxonómicamente por su pequeño tamaño y el grado de trituración. Aún así, la dieta de $R$. fontaniana en la localidad de Lenga estuvo constituida por 11 tipos de presas, siendo los ítemes más frecuentes Cancer setosus (Molina 1782), megalopas (Caridea) y Alpheidae (Tabla 1). La amplitud de nicho trófico ( $\left.\mathrm{B}^{\prime}=0,32\right)$ estaría indicando que estos pulpos tienen una dieta especializada.

En cuanto a la conducta de alimentación, se observó que los pulpos se acercaban lentamente a las presas. Cuando las presas correspondían a crustáceos decápodos, se lanzaban encima envolviéndolas con la umbrela y capturándola con los brazos (Fig. 1a,b). Luego, las presas
Tabla 1

\section{Presas encontradas en el contenido estomacal de $\mathbf{2 0}$ ejemplares de Robsonella fontaniana en Lenga}

Prey items found in the stomach content of 20 specimens of Robsonella fontaniana at Lenga

\begin{tabular}{lcr}
\hline & Frecuencia & $\%$ \\
\hline Teleostei & 2 & 10 \\
Nereididae & 1 & 5 \\
Ostracoda & 2 & 10 \\
Amphipoda & 5 & 25 \\
Megalopa Caridea & 11 & 55 \\
Alpheidae & 11 & 55 \\
Cancer setosus & 19 & 95 \\
Pilumnoides perlatus & 6 & 30 \\
Pinaxodes sp. & 2 & 10 \\
Petrolisthes sp. & 3 & 15 \\
Pagurus sp. & 9 & 45 \\
\hline
\end{tabular}

eran llevadas a la boca, momento en el que el cefalotórax de los cangrejos era abierto por los pulpos en su margen ventral (Fig. 1c,d). Durante la ingestión de la presa, los pulpos eliminaron las partes duras, por lo que se encontraron los restos del exoesqueleto de los cangrejos (e.g. caparazón y apéndices), completamente desarticulados y vacíos (Fig. 1c,d), en los acuarios.

En el experimento de selectividad sobre crustáceos y gasterópodos, los pulpos prefirieron alimentarse de crustáceos $\left(\chi_{(1)}^{2}=5,00 ; P=0,025\right)$. Los crustáceos fueron consumidos el $66,7 \%$ de las veces ofrecidas, mientras que los gasterópodos no fueron consumidos ninguna vez ( $0 \%$ ). Entre las especies ofrecidas, el crustáceo $C$. cinereus fue el ítem presa más consumido $\left(\chi_{(4)}^{2}=10,11 ; P=0,039\right.$, Fig. 2a).

En el experimento de selectividad de presas entre crustáceos, los pulpos prefirieron alimentarse de los ítemes presa con mayor movilidad (E. analoga y $C$. cinereus). E. analoga fue consumido en un $75 \%$, $C$. cinereus, en un $33,3 \%$ y $C$. coronatus, en un $16,7 \%$ de las oportunidades en que estas fueron ofrecidas. Los resultados estadísticos mostraron que las frecuencias de alimentación observadas fueron significativamente distintas a la proporción de consumo esperada (1:1 entre todos los ítems presa ofrecidos) $\left(\chi_{(4)}^{2}=22,50, P<0,001\right.$, Fig. 2b). 


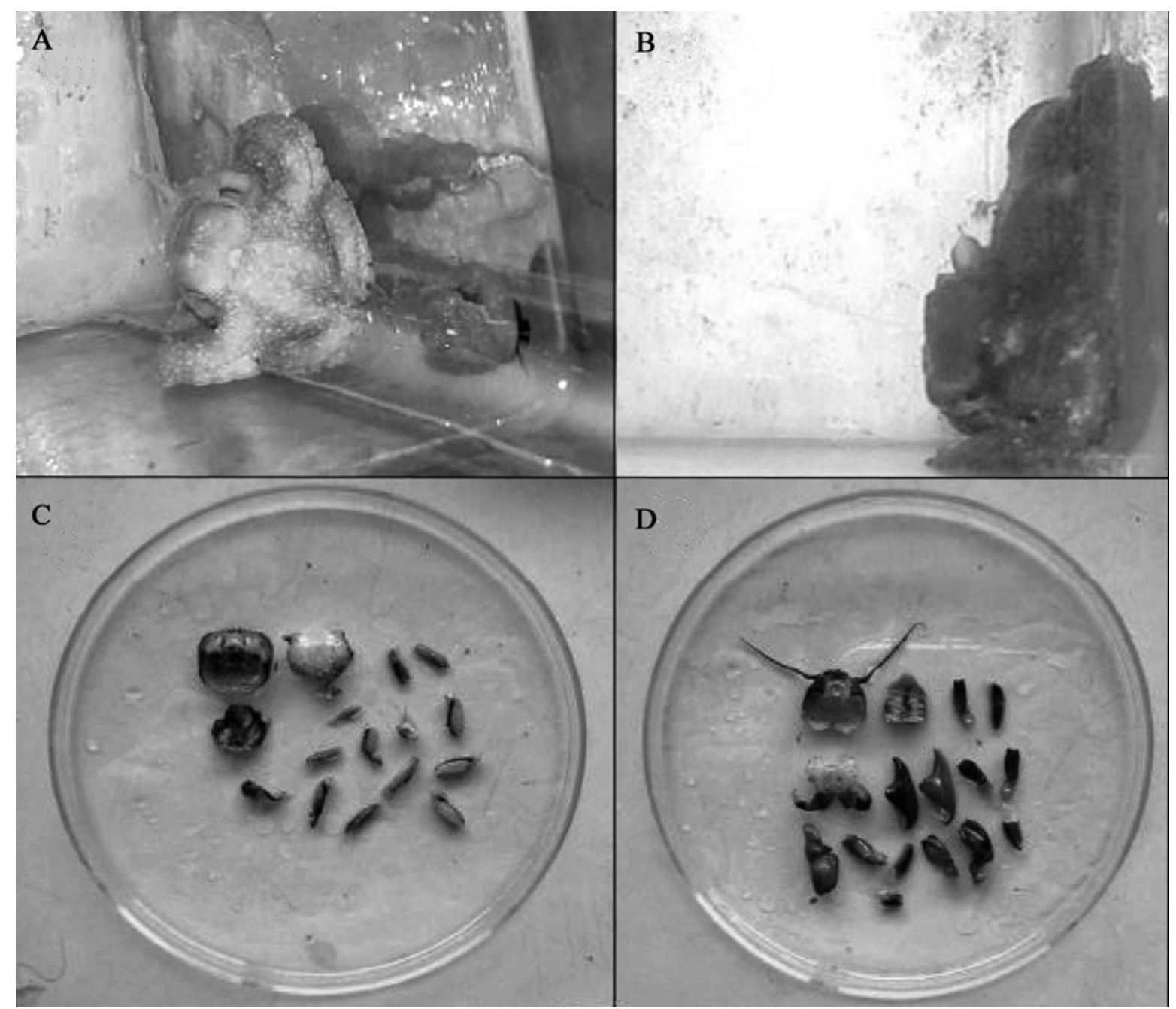

Figura 1

Fotografías de $R$. fontaniana al momento de alimentarse y placas con los restos de presas de las cuales los pulpos se alimentaron

Photographies of $R$. fontaniana during a feeding event and petri dishes with prey remains of which the octopuses were fed

Al relacionar el tamaño de pulpos y presas, se encontró una relación positiva y significativa entre el tamaño de los pulpos y el tamaño de las presas ( $\mathrm{Rs}=0,45$, $\mathrm{n}=26, P=0,027$ ) (Fig. 3a). De la misma forma, el peso total de los pulpos se correlacionó en forma positiva y significativa con el peso del alimento consumido (Rs =
0,81, $\mathrm{n}=16, P=0,001$ ) (Fig. 3b). El peso de alimento consumido en relación al peso corporal de los pulpos varió entre 2,2\% y 15,4\% y a pesar que entre ambas variables se evidenció una tendencia inversa, ésta no fue significativa (Rs $=-0,14, \mathrm{n}=16, P=0,601)$. 


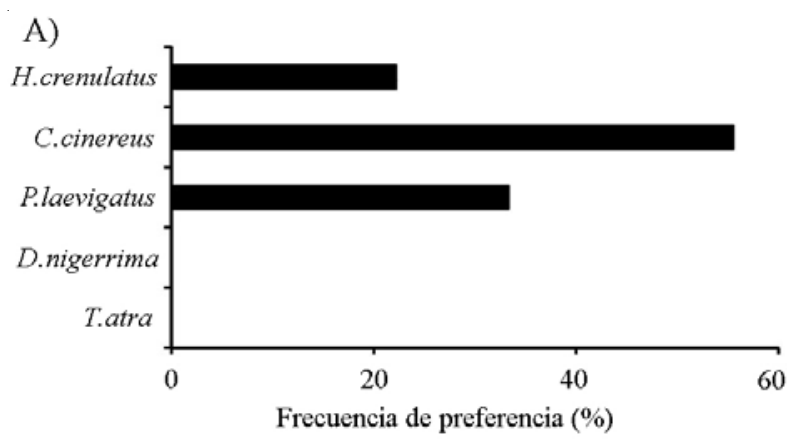

B)

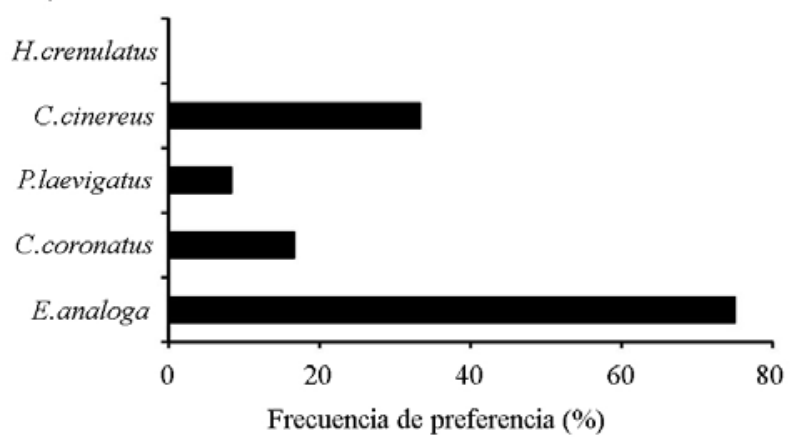

Figura 2

Frecuencia de preferencia de presas potenciales para $R$. fontaniana entre A) crustáceos y gasterópodos $(n=9)$, B) especies de crustáceos $(n=12)$

Preference frequency of potential prey by $R$. fontaniana between A) crustaceans and gastropods $(n=9)$,

B) crustacean species $(n=12)$

\section{Discusión}

Los resultados obtenidos, tanto del contenido estomacal como de los experimentos de preferencias alimenticias, indican que la dieta del pulpo Robsonella fontaniana se compone principalmente de crustáceos decápodos. Varios estudios de campo han revelado que algunas especies de pulpos se alimentan preferentemente de crustáceos (Guerra 1978, Ambrose 1984, Villanueva 1993, Quetglas et al. 1998, Grubert et al. 1999, Oosthuizen \& Smale 2003, Smith 2003, Ibáñez \& Chong 2008) y otras preferentemente de moluscos (Smale \& Bucham 1981, Ambrose 1986, Cortez et al. 1995, Anderson et al. 2008). Sin embargo, se ha descrito que la proporción de los principales ítemes presas (i.e. crustáceos, moluscos y peces) disponibles en el ambiente puede cambiar geográficamente (Rosa et al. 2004). El pulpo Octopus bimaculatus Verrill, 1883 en experimentos de campo y
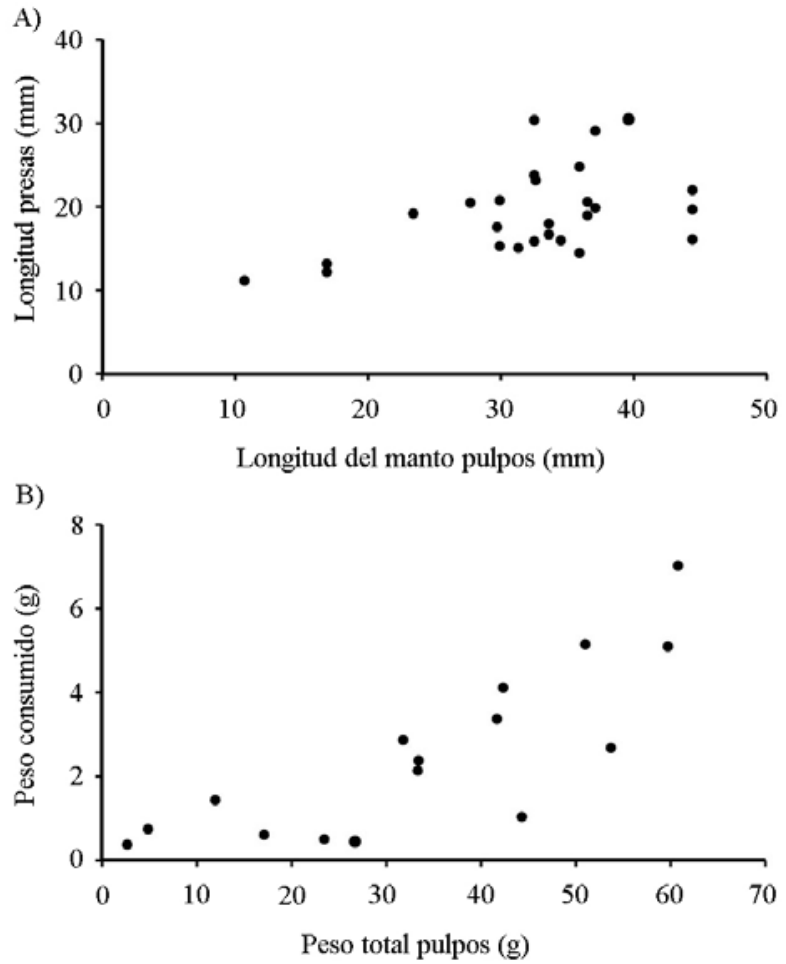

Figura 3

Correlaciones entre A) la longitud del manto de $\boldsymbol{R}$. fontaniana y la longitud de sus presas $(\mathrm{mm})(\mathrm{n}=26)$,

B) el peso total de los pulpos y el peso del alimento consumido $(g)(n=26)$

Correlations between A) $R$. fontaniana mantle length and prey length $(\mathrm{mm})(\mathrm{n}=26)$, B) octopus total weight and consumed food weight $(\mathrm{g})(\mathrm{n}=26)$

laboratorio se alimenta preferentemente de cangrejos y los caracoles fueron los menos preferidos a pesar de que eran los más abundantes en su hábitat (Ambrose 1984). Por otro lado, en experimentos de laboratorio, Pérez et al. (2006) determinaron que las tasas de crecimiento del pulpo Enteroctopus megalocyathus (Gould, 1852) fueron mayores cuando los animales eran alimentados con dietas basadas en crustáceos en comparación con dietas basadas en moluscos mitílidos, los cuales contiene mayor energía. Contrariamente, en otro estudio se ha encontrado que los crustáceos y en particular los decápodos contienen uno de los más altos contenidos calóricos entre los invertebrados marinos, lo cual sugeriría una estrategia de depredación basada en la maximización de la energía (Ojeda \& Dearborn 1991). La preferencia por crustáceos podría ser generada por la ingestión de compuestos necesarios para las moléculas que transportan oxígeno en la hemocianina de los pulpos, los cuales son 
proporcionados por los tejidos de los crustáceos (ver Pérez et al. 2006).

Robsonella fontaniana se alimenta de pocas especies presas, comparado con otras especies de pulpos (e.g. O. bimaculatus $>55$ especies presas, Ambrose 1986), pero se puede decir, a pesar de los pocos estómagos analizados y del índice de amplitud trófica ( $\mathrm{B}^{\prime}=0,32$ ), que posee un nicho trófico reducido, característica por la cual su dieta puede ser clasificada como especializada (sensu Jaksic \& Marone 2007). En la localidad donde fue realizado este estudio (Lenga, Chile), las especies de cangrejos juveniles más abundantes son los decápodos Cancer setosus, Paraxanthus barbiger (Poeppig, 1836), Gaudichaudia gaudichaudi (Milne-Edwards, 1834) y Pilumnoides perlatus (Poeppig, 1836) (Palma et al. 2006), algunos de los cuales corresponden a componentes importantes de la dieta de $R$. fontaniana. Estos datos determinarían que $R$. fontaniana podría presentar una conducta de caza característica de un depredador oportunista. No obstante, los experimentos de preferencias alimenticias en moluscos y crustáceos muestran selectividad sobre las presas y su tamaño. En unos pocos estudios se ha reportado que los pulpos Octopus vulgaris Cuvier, 1797 y O. maorum Hutton, 1880 presentan una conducta de caza combinada entre oportunista y selectiva (Mather 1991, Grubert et al. 1999, Anderson et al. 2008). Sin embargo, para poder verificar correctamente esta hipótesis es necesario realizar estudios complementarios que establezcan diferentes proporciones de presas en experimentos de laboratorio y, además, cuantificar la oferta ambiental de las presas con el objetivo de compararla con el contenido estomacal de los individuos de $R$. fontaniana.

Dentro de los ítems presa ofrecidos a $R$. fontaniana, hubo preferencia por los crustáceos. Particularmente, Emerita analoga fue la especie presa más consumida. Cabe destacar que esta especie se caracteriza por su gran movilidad en fondos blandos del intermareal y submareal somero, que es el hábitat del pulpo $R$. fontaniana (Ibáñez et al. 2008). En general, los cefalópodos son cazadores netamente visuales, los cuales se guían por los movimientos de sus presas (Guerra 1978, Mather 1991, Hanlon \& Messenger 1996), situación que podría estar explicando el consumo preferencial de E. analoga por parte de $R$. fontaniana. Sin embargo, también poseen un desarrollado sentido quimiorreceptor y en experimentos de laboratorio se ha encontrado que los pulpos son atraídos por extractos de cangrejos, algunos aminoácidos, proteínas y moléculas como ATP y AMP (ver Lee 1994). Además, los estímulos quimiotactiles (e.g. textura, forma, tamaño y quimioatractores) son usados para juzgar la palatabilidad de las presas (Lee 1994).
En los experimentos de laboratorio no se observaron evidencias de perforaciones en el exoesqueleto de los crustáceos consumidos por los pulpos. Esto, y en base a los hallazgos de Guerra (1978), sugerimos que la cefalotoxina (glicoproteína tóxica) del pulpo podría estar penetrando a través de las articulaciones o los espacios naturales de las presas, para llegar al sistema nervioso. Debido a que tanto los quelípodos, caparazones y patas de los cangrejos estaban vacías de tejidos posteriormente a la alimentación, se sugiere que la conducta de alimentación de $R$. fontaniana es similar a la observada por otras especies de pulpos (Guerra 1978, Mather 1991, Forsythe \& Hanlon 1997).

A partir de las evidencias de terreno y de laboratorio mostradas en este estudio, se plantea que el pulpo $R$. fontaniana presenta una conducta de caza selectiva, una dieta especializada representada por un nicho trófico restringido y la táctica de caza característica de un maximizador de energía (ver Jaksic \& Marone 2007, Tabla 4.1), la cual no debería ser distinta de otras especies de pulpos. De esta manera, se puede sugerir que la dieta de $R$. fontaniana en la naturaleza se ve influida tanto por las preferencias alimentarias como por la disponibilidad de presas y su calidad energética.

\section{Agradecimientos}

Los autores agradecen los valiosos comentarios de tres revisores anónimos en la mejora del manuscrito. Este trabajo fue financiado por el proyecto 'DIN 08-2003 Biología y conducta de Robsonella fontaniana (d’Orbigny, 1834) (Cephalopoda: Octopodidae) en poblaciones locales de la octava región, Chile'. C.M. Ibáñez y R.D. Sepúlveda agradecen las becas de doctorado CONICYT-Chile.

\section{Literatura citada}

Ambrose RF. 1984. Food preferences, prey availability, and the diet of Octopus bimaculatus Verril. Journal of Experimental Marine Biology and Ecology 77: 29-44.

Ambrose RF. 1986. Effects of octopus predation on motile invertebrates in a rocky subtidal community. Marine Ecology Progress Series 30: 261-273.

Ambrose RF \& BV Nelson. 1983. Predation by Octopus vulgaris in the Mediterranean. Marine Ecology 4: 251-261.

Anderson RC \& JA Mather. 2007. The packaging problem: bivalve prey selection and prey entry techniques of the octopus Enteroctopus dofleini. Journal of Comparative Psychology 121(3): 300-305.

Anderson RC, J Wood \& JA Mather. 2008. Octopus vulgaris in the Caribbean is a specializing generalist. Marine Ecology Progress Series 371: 199-202. 
Cardona L. 1991. Measurement of trophic niche breadth using occurrence frequencies. Journal of Fish Biology 39: 901-903.

Cardoso F, P Villegas \& C Estrella. 2004. Observaciones sobre la biología de Octopus mimus (Cephalopoda: Octopoda) en la costa peruana. Revista Peruana de Biología 11: 45-50.

Cortez T, BG Castro \& A Guerra. 1995. Feeding dynamics of Octopus mimus (Mollusca: Cephalopoda) in northern Chile waters. Marine Biology 123: 497-503.

Fiorito G \& F Gherardi. 1999. Prey-handling behaviour of Octopus vulgaris (Mollusca, Cephalopoda) on bivalve preys. Behavioural Processes 46: 75-88.

Forsythe JW \& RT Hanlon. 1997. Foraging and associated behavior by Octopus cyanea Gray, 1849 on a coral atoll, French Polynesia. Journal of Experimental Marine Biology and Ecology 209: 15-31.

Grubert M, V Wadley \& R White. 1999. Diet and feeding strategy of Octopus maorum in southeast Tasmania. Bulletin of Marine Science 65: 441-451.

Guerra A. 1978. Sobre la alimentación y el comportamiento alimentario de Octopus vulgaris. Investigación Pesquera, España 42: 351-364.

Hammer Ø, DAT Harper \& PD Ryan. 2001. PAST: Paleontological Statistics Software Package for Education and Data Analysis. Palaeontología Electrónica 4: 1-9. [en línea] <http://palaeo-electronica.org/2001_1/past/past.pdf>

Hanlon RT \& JB Messenger. 1996. Cephalopod behaviour, 248 pp. Cambridge University Press, Cambridge.

Ibáñez CM \& J Chong. 2008. Feeding ecology of Enteroctopus megalocyathus (Gould, 1852) (Cephalopoda: Octopodidae). Journal of the Marine Biological Association of the United Kingdom 88: 793-798.

Ibáñez CM, RD Sepúlveda, J Guerrero \& J Chong. 2008. Redescription of Robsonella fontaniana (Cephalopoda: Octopodidae). Journal of the Marine Biological Association of the United Kingdom 88: 617-624.

Iribarne OO, ME Fernández \& H Zucchini. 1991. Prey selection by the small Patagonian octopus Octopus tehuelchus d'Orbigny. Journal of Experimental Marine Biology and Ecology 148: 271-281.

Jaksic FM \& L Marone. 2007. Depredación. En: Jaksic FM \& L Marone (eds). Ecología de comunidades, pp. 69-90, Ediciones Universidad Católica de Chile, Santiago.

Lee PG. 1994. Nutrition of cephalopods: fueling the system. En: Pörtner HO, RK O’Dor \& DL Macmillan (eds). Physiology of cephalopods molluscs: lifestyles and performance adaptations, pp. 35-51, Gordon \& Breach Publishers, Postfach.

Mather JA. 1991. Foraging, feeding and prey remains in middens of juvenile Octopus vulgaris (Mollusca: Cephalopoda). Journal of Zoology, London 224: 27-339.
Ojeda FP \& JH Dearborn. 1991. Feeding ecology of benthic mobile predators: experimental analyses of their influence in rocky subtidal communities of the Gulf of Maine. Journal of Experimental Marine Biology and Ecology 149: 13-44.

Oosthuizen A \& MJ Smale. 2003. Population biology of Octopus vulgaris on the temperate south-eastern coast of South Africa. Journal of the Marine Biological Association of the United Kingdom 83: 535-541.

Palma AT, LM Pardo, R Veas, C Cartes, M Silva, K Manríquez, A Díaz, C Muñoz \& FP Ojeda. 2006. Coastal brachyuran decapods: settlement and recruitment under contrasting coastal geometry conditions. Marine Ecology Progress Series 316: 139-153.

Pérez MC, DA López, K Aguila \& ML González. 2006. Feeding and growth in captivity of the octopus Enteroctopus megalocyathus (Gould, 1852). Aquaculture Research 37: 550-555.

Quetglas A, F Alemany, A Carbonell, P Merella \& P Sánchez. 1998. Biology and fishery of Octopus vulgaris Cuvier, 1797, caught by trawlers in Mallorca (Balearic Sea, Western Mediterranean). Fisheries Research 36: 237-249.

Retamal MA. 1981. Catálogo ilustrado de los crustáceos decápodos de Chile. Gayana Zoología 44: 1-110.

Rodhouse PG \& CM Nigmatullin. 1996. Role as consumers. Philosophical Transactions of the Royal Society of London B 351: 1003-1022.

Rosa R, AM Marques, ML Nunes, N Bandarra \& CS Reis. 2004. Spatial-temporal changes in dimethyl acetal (Octadecanal) levels of Octopus vulgaris (Mollusca: Cephalopoda): relation to feeding ecology. Scientia Marina 68: 227-236.

Smale MJ \& P Buchan. 1981. Biology of Octopus vulgaris off the East Coast of South Africa. Marine Biology 65: 1-12.

Smith CD. 2003. Diet of Octopus vulgaris in False Bay, South Africa. Marine Biology 143: 1127-1133.

Steer MA \& JM Semmens. 2003. Pulling or drilling, does size or species matter? An experimental study of prey handling in Octopus dierythraeus (Norman, 1992). Journal of Marine Biology and Ecology 290: 165-178.

Villanueva R. 1993. Diet and mandibular growth of Octopus magnificus (Cephalopoda). South African Journal of Marine Science 13: 121-126.

Vincent TLS, D Scheel \& KR Hough. 1998. Some aspect of the diet and foraging behaviour of Octopus dofleini (Wulker, 1910) in its northernmost range. Marine Ecology 19: 13-29.

Zar JH. 1999. Biostatistical analysis, 663 pp. Prentice Hall, Englewood Cliffs. 